\title{
Miniaturised photonic front-end for the next generation of space SAR applications
}

T. Mengual, Vanessa Duarte, V. Polo, M. Piqueras, P. Sanchis, et al.

T. Mengual, Vanessa C. Duarte, V. Polo, M. A. Piqueras, P. Sanchis, A. Brimont, D. Zurita, B. Chmielak, S. Suckow, A. Giesecke, E. Matarazzo, L. DiPalma, D. Maiarelli, H. Wang, P. G. Huggard, "Miniaturised photonic frontend for the next generation of space SAR applications," Proc. SPIE 11852, International Conference on Space Optics - ICSO 2020, 1185255 (11 June 2021); doi: 10.1117/12.2599928

SPIE Event: International Conference on Space Optics - ICSO 2021, 2021, Online Only 


\section{International Conference on Space Optics-ICSO 2020}

Virtual Conference

30 March-2 April 2021

Edited by Bruno Cugny, Zoran Sodnik, and Nikos Karafolas
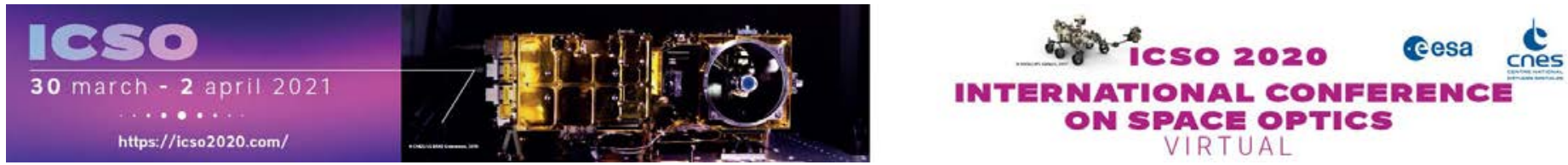

\section{Miniaturised photonic front-end for the next generation of space SAR applications}

\section{Cesa isoporecestings denes}




\title{
Miniaturised photonic front-end for the next generation of space SAR applications
}

\author{
T. Mengual ${ }^{* a}$, Vanessa C. Duarte ${ }^{\mathrm{a}}$, V. Polo ${ }^{\mathrm{a}}$, M. A. Piqueras ${ }^{\mathrm{a}}$, P. Sanchis ${ }^{\mathrm{b}}$, A. Brimont ${ }^{\mathrm{b}}$, D. Zurita ${ }^{\mathrm{b}}$, B. \\ Chmielak $^{\mathrm{c}}$, S. Suckow ${ }^{\mathrm{c}}$, A. Giesecke ${ }^{\mathrm{c}}$, E. Matarazzo ${ }^{\mathrm{d}}$, L. DiPalma ${ }^{\mathrm{d}}$, D. Maiarelli ${ }^{\mathrm{d}}$, H. Wang ${ }^{\mathrm{e}}$ and P. G. \\ Huggarde. \\ ${ }^{a}$ DAS Photonics, Camino de Vera, s/n Edificio 8F, 46022 Valencia, Spain

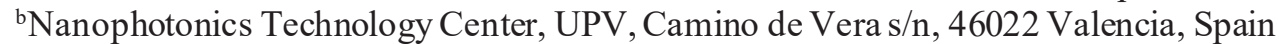 \\ ${ }^{\mathrm{c}} \mathrm{AMO} \mathrm{GmbH}$, Otto-Blumenthal-Str. 25,52074 Aachen, Germany \\ ${ }^{\mathrm{d}}$ AIRBUS Italia S.p.A., Via dei Luxardo 22-24 - 00156 Rome, Italy \\ eRAL Space, STFC Rutherford Appleton Laboratory, Ox11 0QX, Didcot, UK
}

\begin{abstract}
There is a naturaltrade-off between spacecraft size and functiona lity in all current satellite applications, indepen dently of orbit and mission. Therefore, advances in both miniaturization and integration technologies are required to increase satellites' lifetime and performance, simultaneously reducing their cost. In case of the next generation of Earth Observation satellites, one of the key development areas is synthetic aperture radar (SAR) antennas, where expected progress will be to increase the operating bandwidth - requiring, for instance wideband true-time delay (TTD) beamformers - and minia turization, drastically reducing the mass and volume compared to current implementations.

In this scenario, the use of photonic integrated circuits (PIC) technology in the beamforming network, in combination with an optical fibre harness, are obvious key enabling technologies for future SAR instruments. Optically implemented TTD beamforming structures achieve orders-of-magnitude improvements in size and mass compared with coaxial cable and RF switch based alternatives. Photonic technology also brings easy routing thanks to wavelength-division multiplexing, antenna and RF system integration due to the EMI-free characteristic of the optical fibre and a reduction of the risks associated with the in-orbit antenna deployment. Additionally, the inherent broadband characteristic of phot onic technology, related to the transport and processing of RF signals, simplifies the beamforming network and signal distribution design for different frequencies, applications and missions.

In the H2020 RETINA project (H2020-SPACE-2018-821943) a consortium formed by DAS Photonics, Airbus Italia, AMO GmbH, STFC Rutherford Appleton Laboratory and Universitat Politècnica de València is developing a miniaturised photonic front-end for next-generation X-band space SAR applications. In this article we present advances in design and fabrication of PIC for TTD, the design and predicted performance of multi element, dual polarisation antenna building blocks and photoreceivers for phase and a mplitude controlled optical to RF conversion.
\end{abstract}

Keywords: Synthetic Aperture Radar (SAR), microwave photonics, optical beamforming, phased-array antennas, photonic integrated circuit (PIC), True-Time Delay

\section{INTRODUCTION}

In recent years small satellites have become more attractive due to lower development costs and shorter lead times. There is a naturaltrade-off between spacecra ft size and functiona lity in all current satellite applications, independently of orbit and mission, but advances in both miniaturization and integration technologies have diminished the scope of that trade-off. The obvious development requirement for SAR is to increase the operative bandwidth and miniaturization of the antenna. The miniaturization will drastically reduce the mass and volume, including the RF system, when compared with current achievements.

*tmengual@dasphotonics.com; phone +34 963556 150; fax +34 963562 581; http://www.dasphotonics.com/ 
Increasing the SAR frequency helps to achieve this target, but at the same time it introduces new difficulties: a shorter wavelength can negatively affect the coherence of the interferometric signal, the maximum terra in velocity measura ble, and brings some difficulties in the management of atmospheric artefacts. However, these negative effects are compensated by the higher spatial resolution and a shorter revisit time that could be achieved by the use of transmitted chirp with larger frequency bandwidth ${ }^{1}$.

The evolution of SAR has shown a clear trend towards systems with improved performance resulting in higher complexity (larger antennas, operating bandwidth and/or different frequencies) at lower cost, less mass, size and power consumption ${ }^{2}$. This trend imposes strong requirements on today's RF and antenna technology since larger antennas means a complex, bulky and difficult to route RF harness to transport the signal from/to the beamforming network to/from the antenna. There a re accompanying demanding mechanical and thermal requirements, especially when in-orb it deployable antenna structures are required. On the other hand, larger bandwidths associated with larger antennas and scanning angles requires true-time-delay (TTD) beamforming, resulting in bulky and complex solutions (and very limited for lower frequencies / larger delays) impacting directly in the size, mass and integration cost.

In this scenario, the use of photonic integrated circuits (PIC) technology in the beamforming network, in combination with an optical fibre harness, are obvious key enabling technologies for future SAR instruments. Optically implemented wideband TTD beamforming structures achieve orders-of-magnitude improvements in size and mass. Photonic technology also brings easy routing thanks to wavelength-division multiplexing, antenna and RF system integration due to the EMI-free characteristic of the optical fibre and a reduction of the risks associated with the in-orbit antenna deployment. Additionally, the inherent broadband characteristic of photonic technology, related to the transport and processing of RF signals, simplifies the beamforming network and signal distribution design for different frequencies, applications and missions. This is because the same physical media can be used, as opposed to traditional RF implementations in which different substrates, waveguides and components must be used for different frequencies of operation.

In the frame of the H2020 RETINA project (H2020-SPACE-2018-821943) a consortium formed by DAS Photonics, Airbus Italia, AMO GmbH, STFC Rutherford Appleton Laboratory and Universitat Politècnica de València is developing a miniaturised photonic front-end for next-generation space SAR applications. In particular, the main objectives of the RETINA project are:

- Development of an advanced reconfigurable multi-beam photonic beamformer with centralised processing capable of generating up to 256 beams and feeding an array antenna with up to 256 elements.

- The development of a frequency-a gnostic, TTD beamforming network PIC with capability of 64 simultaneous beams and 64 antenna elements in a $2 \times 2 \mathrm{~cm}^{2}$ device implemented in $\mathrm{Si}_{3} \mathrm{~N}_{4}$ technology, to a chieve a $256 \times 256$ beamformer.

- Design and manufacturing to achieve TRL 6 of:

- A dual polarisation array antenna element design at X-band (8 -12 GHz)

- A broadband beam optical transmitter module, including RF, optical amplification and modulation, reducing size and footprint.

- A compact X-band antenna optical receiver with a co-packaging of amplifiers, optical receiver and antenna element, reducing the size and footprint of the present solutions.

- Design, manufacturing and supply of photoreceivers in the X-band with optimized footprint and self-stabilized gain vs temperature.

- Demonstration of the flexibility of beam-shaping and beam-switching in a PIC-based optical beamformer by the combination of a fixed beamformer network, generating multiple orthogonal beams, with a co-integrated switching network to perform beam-shaping by multi-orthogonal beam synthesis. 
The paper discusses the application scenario for the technology in terms of three SAR mission concepts and present technicaladvances towards these objectives in three areas:

- Design of X-band photoreceivers with programmable ga in and phase control

- PIC a rchitecture; design and test of building blocks for the PIC

- Design and predicted performance of dual polarization antenna elements

\section{APPLICATION SCENARIO}

In this section some missions are proposed that could benefit from the technology development discussed in this paper. The missions are based on reduction of mass and volume achievable with PIC TTD technology, which could make it possible to fly a SAR instrument on small platforms. The approach is in line with the actual trend to reduce the mission cost and development time and on the possibility of mission in flight formation, interest for which is considerably increased in Europe and in the United States.

Formation flight missions can provide results not achievable with a single satellite, both in the scientific and application services but necessarily requires the development of spacecrafts of reduced mass and cost, possibly compatible with multiple launches for a further cost reduction of the overall mission. The potential application for formation flight missions are interferometry and missions involving bi-static SAR, with one transmit/receive SAR and one or more receive only systems. A further advantage of formation flight mission is that of the reconfigurability and therefore to adapt the mission to different applications during the mission lifetime.

Table 1. Reference requirements for the proposed application scenario missions.

\begin{tabular}{|c|c|c|c|}
\hline Parameter & $\begin{array}{c}\text { Mission \#1 } \\
\text { (small platforms) }\end{array}$ & $\begin{array}{l}\text { Mission \#2 } \\
\text { (satellites in } \\
\text { formation) } \\
\end{array}$ & $\begin{array}{c}\text { Mission \#3 } \\
\text { (large platforms) }\end{array}$ \\
\hline $\begin{array}{l}\text { Operating } \\
\text { frequency }\end{array}$ & X-band, $9.6 \mathrm{GHz}$ & X-band, $9.6 \mathrm{GHz}$ & X-band, $9.6 \mathrm{GHz}$ \\
\hline Signal bandwidth & $500 \mathrm{MHz}$ & $400 \mathrm{MHz}$ & $800 \mathrm{MHz}$ \\
\hline $\begin{array}{c}\text { Relative Signal } \\
\text { Bandwidth }\end{array}$ & $5.2 \%$ & $4.2 \%$ & $8.3 \%$ \\
\hline $\begin{array}{c}\text { Instrument Mass } \\
\text { (including } \\
\text { antenna) }\end{array}$ & $30 \mathrm{~kg}$ & $30 \mathrm{~kg}$ & $250 \mathrm{~kg}$ \\
\hline $\begin{array}{c}\text { Peak Transmitted } \\
\text { Power } \\
\end{array}$ & $1 \mathrm{~kW}$ & $30 \mathrm{~W}$ & $2.8 \mathrm{~kW}$ \\
\hline Access Area & $20^{\circ}-45^{\circ}$ & $20^{\circ}-45^{\circ}$ & $15^{\circ}-55^{\circ}$ \\
\hline Spatial Resolution & $1 \mathrm{~m}-3 \mathrm{~m}$ & $1 \mathrm{~m}-3 \mathrm{~m}$ & $0.5 \mathrm{~m}$ \\
\hline Swath width & $5 \mathrm{~km}-8 \mathrm{~km}$ & $5 \mathrm{~km}-8 \mathrm{~km}$ & $5 \mathrm{~km}-8 \mathrm{~km}$ \\
\hline \multirow{2}{*}{$\begin{array}{c}\text { Noise Equivalent } \\
\sigma 0\end{array}$} & -20dB@1m res. & \multirow{2}{*}{$-25 \mathrm{~dB}$} & \multirow{2}{*}{$-15 \mathrm{~dB}$} \\
\hline & $-30 \mathrm{~dB} @ 3 \mathrm{~m}$ res. & & \\
\hline
\end{tabular}

Three missions are proposed: Table 1. Mission \#1 is a single mode SAR of medium performance which would allow the development of the mission in a short time minimizing the risks and the costs, but maintain ing a good trade-off between the spatial resolution and the image quality. Mission \#2 is a bi-static SAR approach, which can be conveniently used for interferometry. The bi-static approach foresees the physical separation between the transmitter and the receiver. As baseline two platforms are considered: the former hosting a transmit/receive instrument and the latter a receive one. Its main characteristics are reported in the table 1. Finally, Mission \#3 foresees a more ambitious mission embarking a highperformance SAR instrument, to be developed for the acquisition of high resolution images. The mission foresees different SAR operating modes: strip map, spot etc. 


\section{MINIATURISED PHOTONIC FRONT-END ARCHITECTURE DESCRIPTION}

The main objective of this project is the development of compact, suitable for space, broadband frequency operation multi-beam photonic beamformer with centra lized processing for next generation SAR.

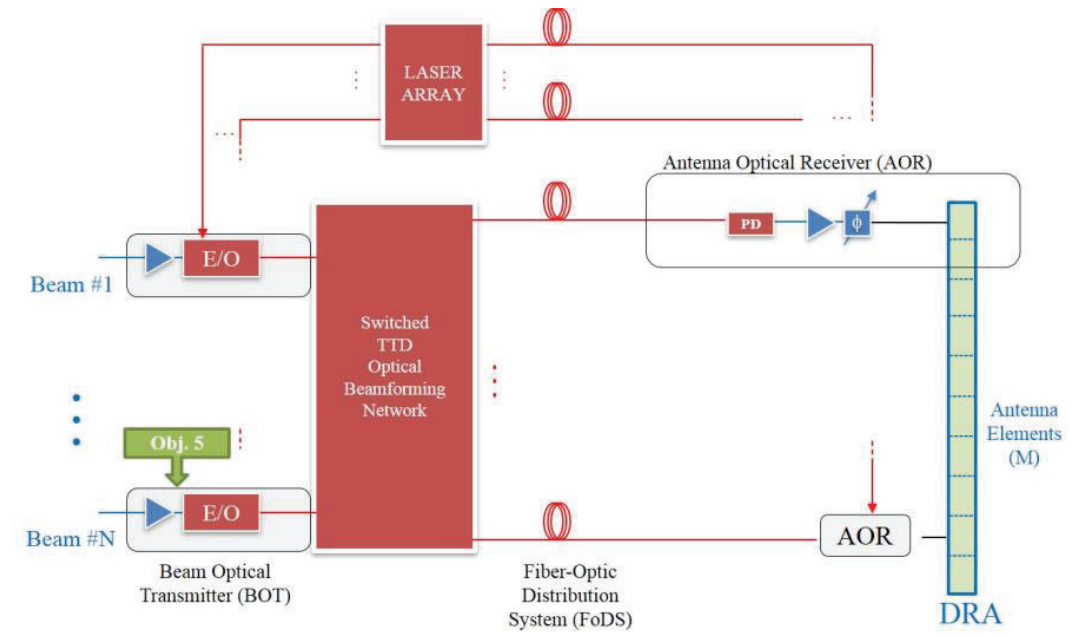

Figure 1. Scheme of the photonic beamformer. RF elements are shown in blue, optical in red.

Figure 1 shows the scheme of multi-beam photonic beamformer which is a sub-populated demonstrator sufficient to assess the main technical functionalities, especially the RF to optical (E/O) conversion, a PIC implementing TTD as basic block of the optical Beamforming Network (BFN), optical to RF (O/E) conversion, a ssociated optical distribution and antenna elements.

It consists of a Direct Radiating Array (DRA) Antenna System composed by several antenna elements that are all fed by the same signals, but with differences in delay and amplitude. The instrument's beam can be focused or "steered" to a predetermined direction by appropriate control of the delays incurred by the signals arriving at the antenna elements. This delay control is performed by a dividing and delaying structure named the beamforming network. This network becomes very complex if the number of elements in the antenna is large. The complexity is usually reduced by using phase control instead of true time delay although this implementation limits the operation bandwidth of the antenna. Phase control means that the beam steers with frequency - an effect named beam squint - and is not suitable for bandwidths larger than a round $250 \mathrm{MHz}$.

The complexity of the beamforming network increases exponentially if a number of simultaneous beams (requiring different signals with independent delay and a mplitude control) are radiated by the sa me antenna, becoming impra ctica 1 for a relatively low number of beams with the present RF technology. In this paper, it is described overcoming this limitation by the use of PIC technology in the beamforming network.

In particular, it is proposed to feed the DRA via optical fibre connecting all the antenna elements with a central signal distribution unit which is in charge of converting the RF signal to be transmitted by the antenna to the optical domain and to provide the required delays to each antenna element. In the antenna optical receiver, the modulated optical sign a ls are converted back to RF by photoreceivers since, the elementary antenna will be co-packaged with a receiver module containing a photodetector and RF amplification. Therefore, each array antenna element will have optical interfaces to connect the optical fibres linking the beamformer network and the antennas.

The photoreceiver integrates a broadband photodiode, low noise amplification and phase and amplitude control. This photoreceivers will be fabricated for $\mathrm{X}$ band with a minimum bandwidth of $600 \mathrm{MHz}$ and a goal of $1.2 \mathrm{GHz}$. The prototype photoreceiver module is shown in Figure 2. 


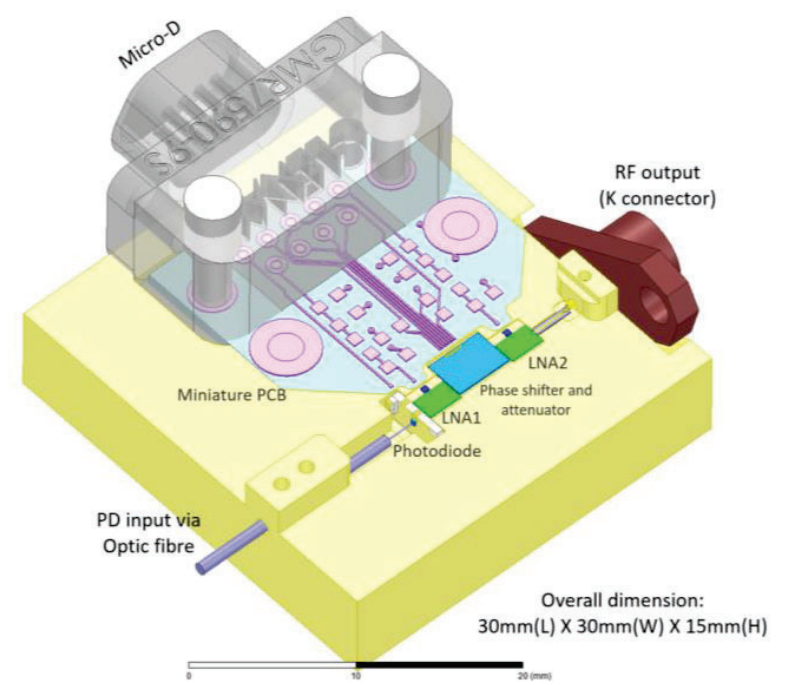

Figure 2. Prototype of the X-band photoreceiver module, performing the optical to RF conversion function.

\section{PHOTONIC INTEGRATED CIRCUIT IMPLEMENTING TRUE-TIME DELAY LINES}

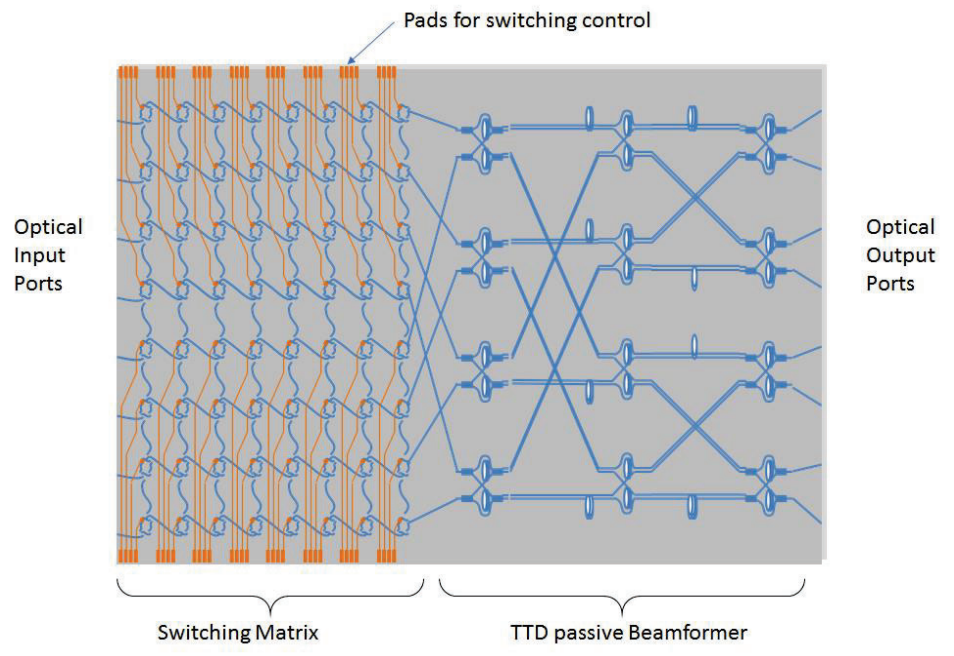

Figure 3. Elementary PIC-based 8x8 beamforming network

The proposed optical beamforming network with TTD characteristic is constructed by aggregating elementary PIC-based $8 \times 8$ beamforming networks with switching capabilities. This unitary photonic chip is depicted in Figure 3 . This component can control the delay of up to eight signals simultaneously and to route and combine them to a specific beamforming port. The $3 \mathrm{D}$ aggregation of this elementary unit is performed to construct the complete SAR beamformer able to steer the beam in any direction within the coverage limits. A simplified scheme of this $3 \mathrm{D}$ aggregation and how the PICs are connected to the antenna by opticalfibre is shown in Figure 4.

In order to fulfil the high technical demands of the surrounding system, especially the low insertion loss, a silicon nitride $\left(\mathrm{Si}_{3} \mathrm{~N}_{4}\right)$ technology for ultra low loss waveguides is employed ${ }^{3,5}$. The waveguide cross section is sketched in Figure 5(a). In this approach the waveguide core consists on a $2.8 \mu \mathrm{m}$ wide and $100 \mathrm{~nm}$ high $\mathrm{Si}_{3} \mathrm{~N}_{4}$ waveguide, located on top of a 6 
$\mu \mathrm{m}$ thick silicon dioxide $\left(\mathrm{SiO}_{2}\right)$ buffer layer that separates it from the silicon ( $\left.\mathrm{Si}\right)$ substrate. The waveguide core is covered by a $1.6 \mu \mathrm{m}$ thick low temperature $\mathrm{SiO}_{2}$ (LTO) cladding layer. Moreover, a metallic layer is deposited on top of the optical waveguide for implementing micro-heaters, required to build thermo-optical switches. The simulated mode profile in the waveguide for TE polarization is shown in Figure 5(c).

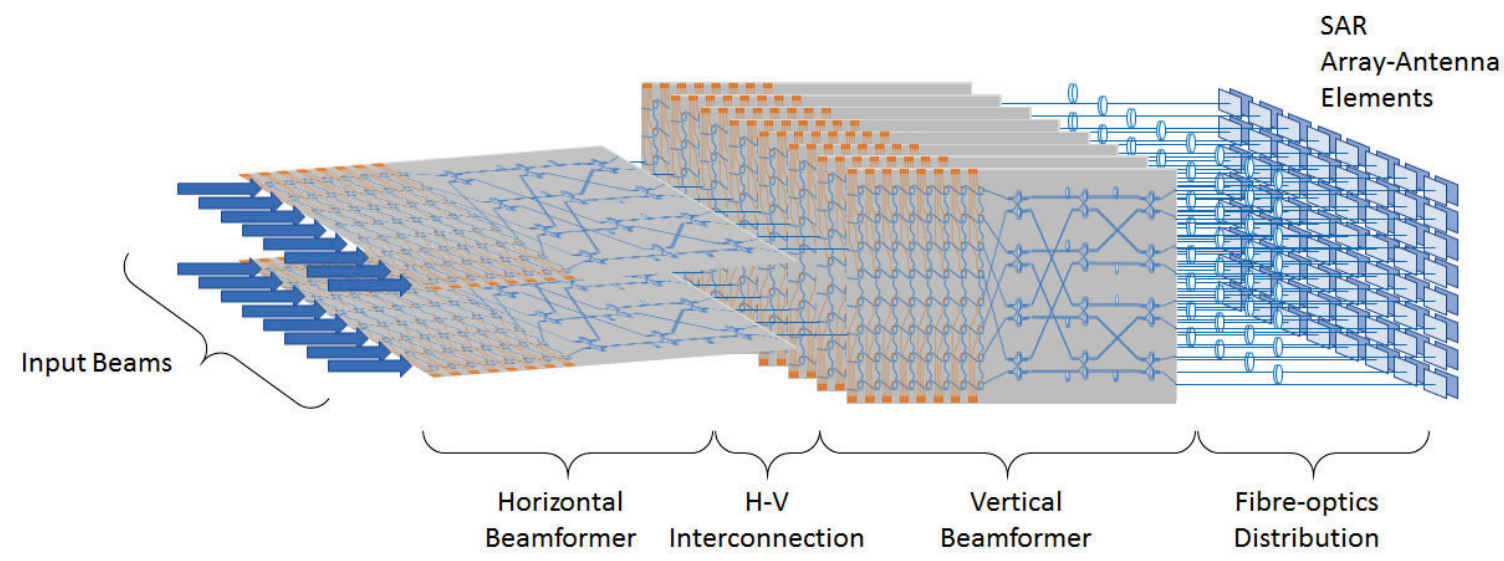

Figure 4. 3D aggregation of elementary $8 \times 8$ beamforming network PICs to construct the complete SAR beamformer

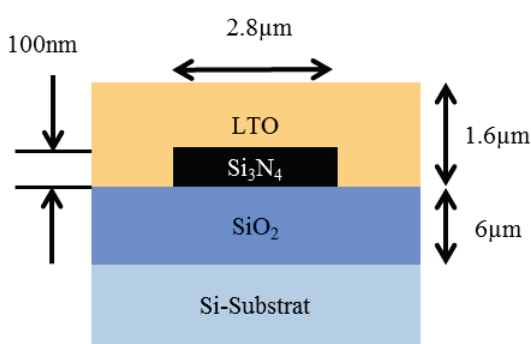

(a)

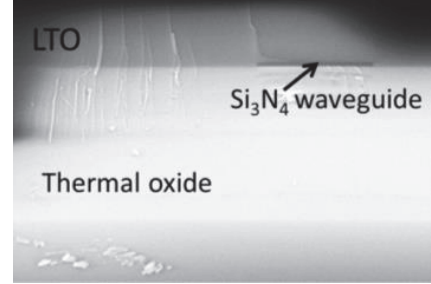

(b)

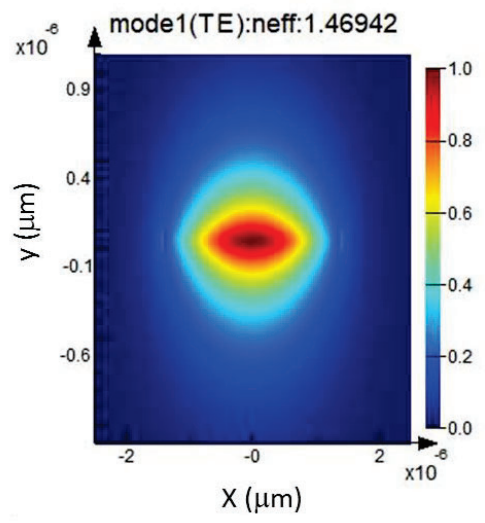

(c)

Figure 5. (a) Ultra-low loss photonic waveguide cross-section, (b) micrograph of fabricated waveguide and

(c) simulated mode profile for TE polarization and 
In order to investigate experimentally the propagation loss the cut-back method has been utilized. For this, a layout with 8 similar waveguides, which have identical coupling structures but differ in length, has been designed and fabricated. The length of the waveguides varies between $1.5 \mathrm{~cm}$ and $107 \mathrm{~cm}$. This large length difference is necessary for a precise measurement of very small propagation losses. A linear fit to the measured transmission at a wavelength of $1550 \mathrm{~nm}$ yields a propagation loss of $0.09 \mathrm{~dB} / \mathrm{cm}$ (Figure 6). The intercept with the y-axis yields a loss of $5.2 \mathrm{~dB}$ per fibre-chip coupler.

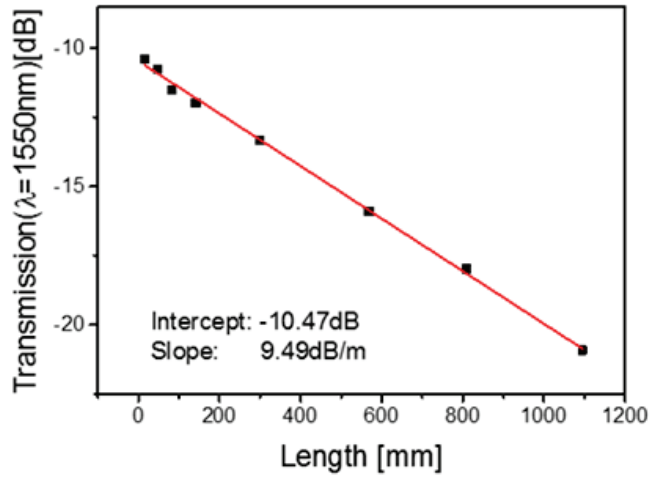

Figure 6. Linear fit to the measured transmission at a wavelength of $1550 \mathrm{~nm}$. Fitted line, red, has a slope of $9 \mathrm{~dB} / \mathrm{m}$.

An efficient fibre-to-chip assembly process is necessary since a high number of input/output (I/O) ports are required for supporting advanced multi-beam operation for space applications. Therefore, an efficient fibre-to-chip assembly process has been developed ${ }^{4}$ and the coupling efficiency has been significantly improved from approximately $10 \mathrm{~dB} /$ coupler down to an average of around $6 \mathrm{~dB} /$ coupler $^{5}$.

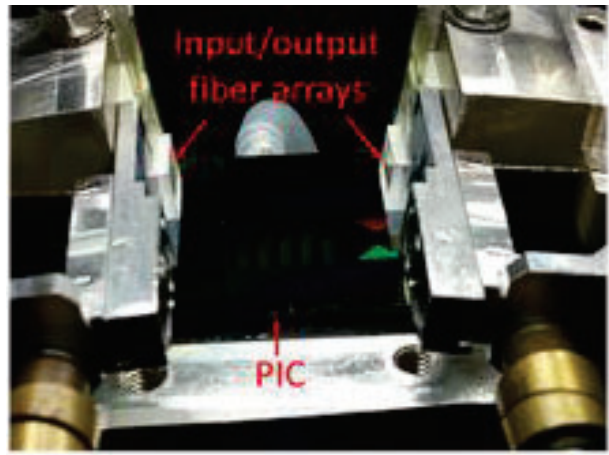

(a)

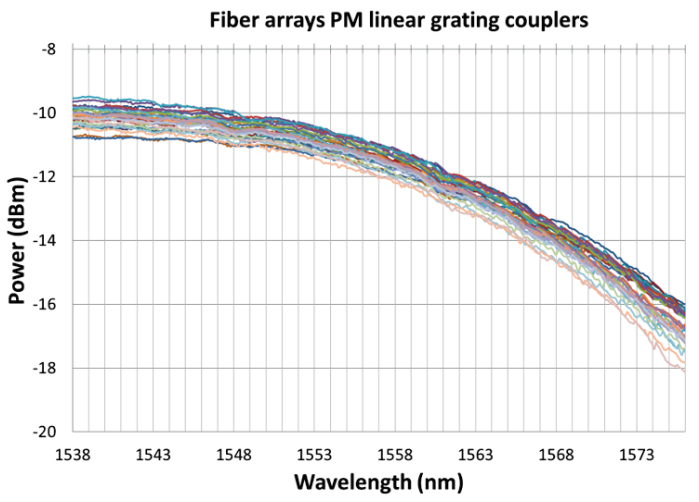

(b)

Figure 7. (a) Picture of the alignment bench after I/O fibre/PIC alignment and (b) spectral transmissions with Polarization maintaining (PM) fibre-arrays (trough the $32 \mathrm{x} \mathrm{I} / \mathrm{O}$ ports)

Figure 7(a) shows an optical microscope image of the PIC fabricated to test coupling and propagation losses. The measured spectra from fibre-to-fibre between different ports are depicted in Figure 7(b). Total fibre-to-fibre insertion losses of around $11.9 \mathrm{~dB}$ and $13.2 \mathrm{~dB}$ were measured, yielding $\approx 5.85$ to $6.6 \mathrm{~dB}$ coupling losses per coupler.

The different photonic integrated building blocks (BB) required to implement the elementary PIC have been designed using the different simulation tools that Lumerical ${ }^{6}$ software offers (e.g. 3D propagation method, 3D eigenmode expansion and FDTD simulations) and tested. The main drivers of the BB design have been:

- To reduce the size of the required photonic integrated building blocks as much as possible

- To provide a broadband PIC in terms of wavelengths to be suitable for a multi-beam scenario 
Figure 8 shows the layout and a picture of the manufactured BB PIC and, as an example, Figure 9 shows the multim ode interference (MMI) $3 \mathrm{~dB}$ coupler design and test results. This BB PIC incorporates:

- $\quad$ MMI $3 \mathrm{~dB}$ couplers

- Focused grating couplers with different period, taper length and fill factor

- Linear grating couplers with different period and fill factor

- Cross angles between waveguides to mea sure the crosses crosstalk

- Pre-a lignment photonic structures for seamless fibre array coupling

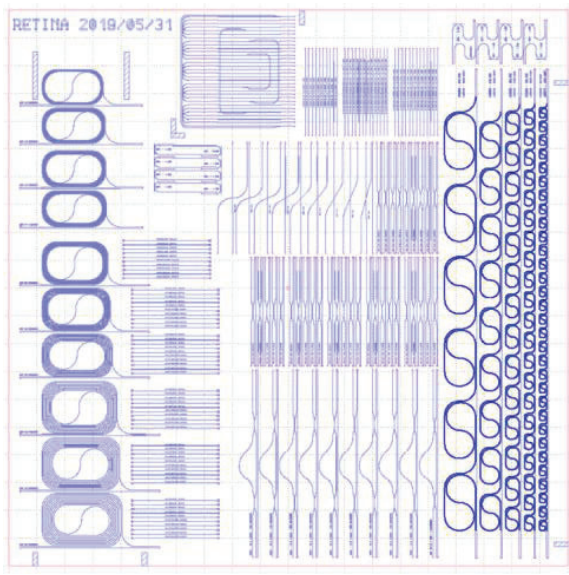

(a)

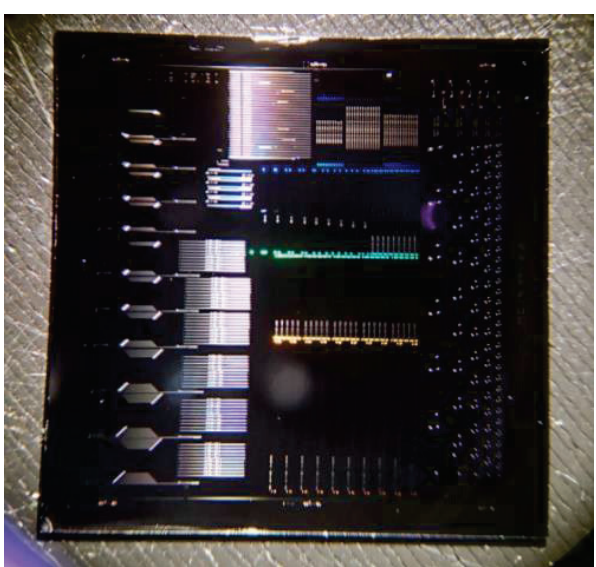

(b)

Figure 8 (a) BB layout and (b) microscope picture of the manufactured BB chip.

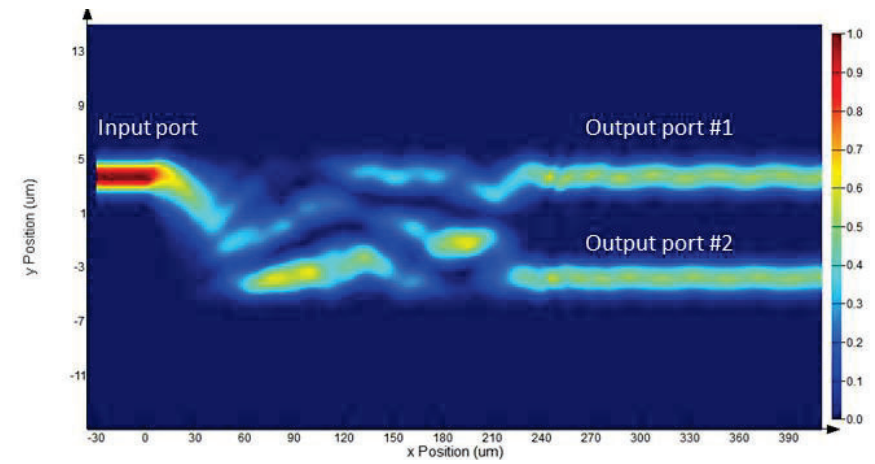

(a)

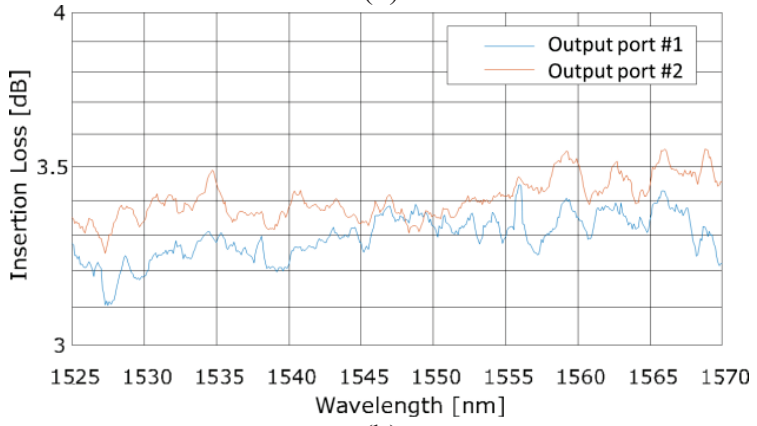

(b)

Figure 9 (a) Simulated E-field density of an implemented multimode interference (MMI) $3 \mathrm{~dB}$ coupler, at a wavelength of $1550 \mathrm{~nm}$ and (b) insertion loss of the MMI measured at different wavelengths. 


\section{SUB-ARRAY ANTENNA ELEMENT}

The other part of the project's SAR design activity is based on the development of an antenna array module in X band, whose electrical requirements will be satisfied over the whole operating frequency range. The main antenna requirements are presented in Table 2 .

Table 2. SAR Antenna panel requirements.

\begin{tabular}{|l|c|l|}
\hline Parameter & Value & Remarks \\
\hline Operative Frequency & X-band $(9.6 \mathrm{GHz})$ & - \\
\hline Frequency Bandwidth & $600 \mathrm{MHz}$ & - \\
\hline Polarization & Dual orthogonallinear(H, V) & $\begin{array}{l}\text { Separated ports for each } \\
\text { polarization }\end{array}$ \\
\hline $\begin{array}{l}\text { Sub-array } \\
\text { Configuration }\end{array}$ & $25.5 \mathrm{~mm}$ & Between 8 and 12 elements \\
\hline Element Spacing & $21.5 \mathrm{~mm}$ & $0.85 \lambda_{0} @ 9.9 \mathrm{GHz}$ \\
\hline Subarray Spacing & $>18 \mathrm{~dB}$ (Target) & $0.70 \lambda_{0} @ 9.9 \mathrm{GHz}$ \\
\hline Return Loss & $<1.6 \mathrm{~dB}$ (Target) & $\begin{array}{l}\text { Radiating element: } 0.35 \mathrm{~dB} \\
\text { Sub-array BFN: } 1.25 \mathrm{~dB}\end{array}$ \\
\hline Insertion Loss & Compatible with SAR mission & 1 W at single radiating element level \\
\hline Power handling & & Lin typical \\
\hline
\end{tabular}

The design of the single radiating element represents a key aspect of the overall antenna since it determines the radiation performance of the antenna. The selection of the technology to realise it has a direct impact on the other part of the design (e.g. transitions, BFN integration, etc.).

Several configurations have been investigated in order to select the best single radiating element architecture. In addition to the requirements summarized in Table 2 , the main drivers of the design have been:

- Low-profile architecture. Since the SAR application require thousands of radiating elements, a solution characterized by reduced mass and compact size is mandatory.

- Suitable for BFN integration. Each radiating element a rray is formed by different radiating elements. These elements should be developed in a technology compatible with compact BFNs.

- Reduced manufacturing costs. This is necessary to minimise the effective costs of the whole SAR antenna panels.

The developed Antenna Element Sub-array (AES) is formed by eight single radiating elements and two different BFNs, one for each polarization. A 3D view of the AES model is shown in Figure 10.

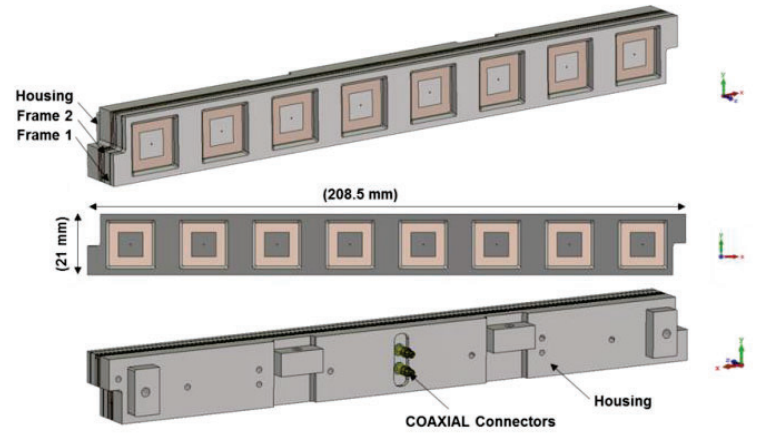

Figure 10. 3D sketch of the AEA 
The AES's preliminary RF performance has been verified by a proper set of full-wave simulations. The predicted reflection coefficient and isolation between $\mathrm{H}$ and $\mathrm{V}$ ports is reported in Figure 11. Simulated radiation patterns of the AES with integrated dual-polarized BFN are reported in Figure 12 for both $\mathrm{H}$ and V ports. Finally, the predicted overall loss, including ohmic and dielectric losses, is lower than $1.55 \mathrm{~dB}$.

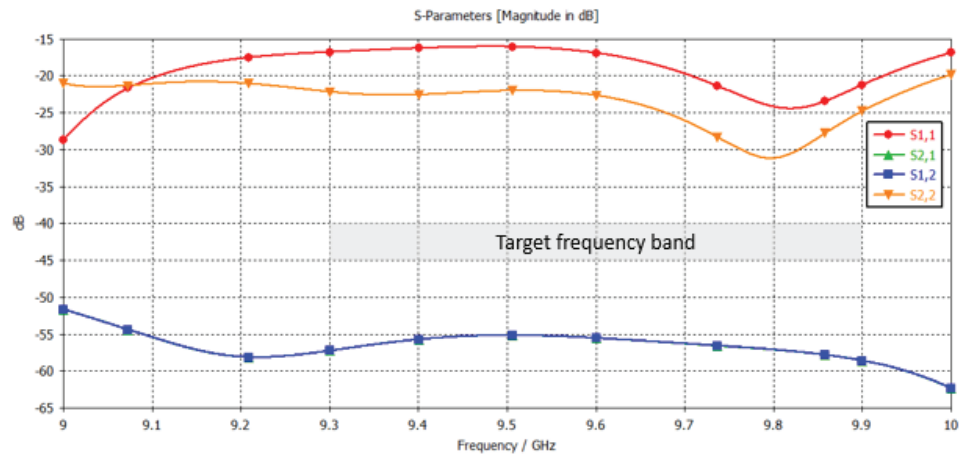

Figure 11. Simulated input reflection coefficients at port $\mathrm{H}$ and port $\mathrm{V}$ and isolation between them of the AES shown in Figure 10.

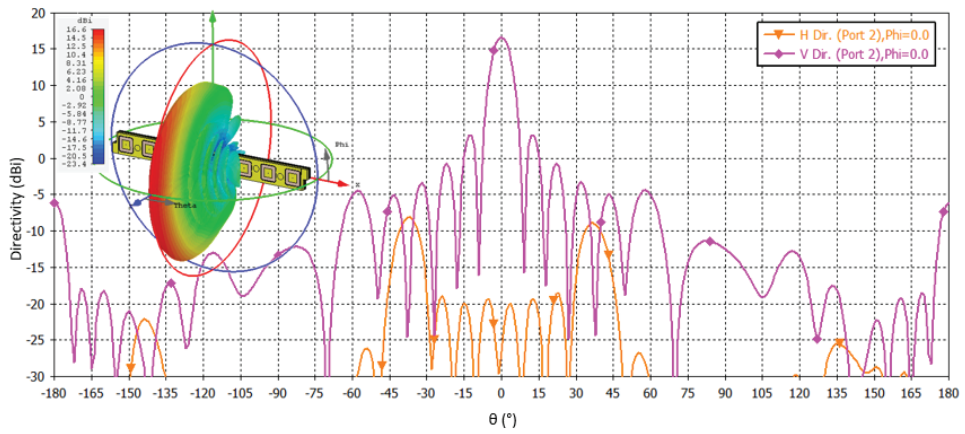

(a)

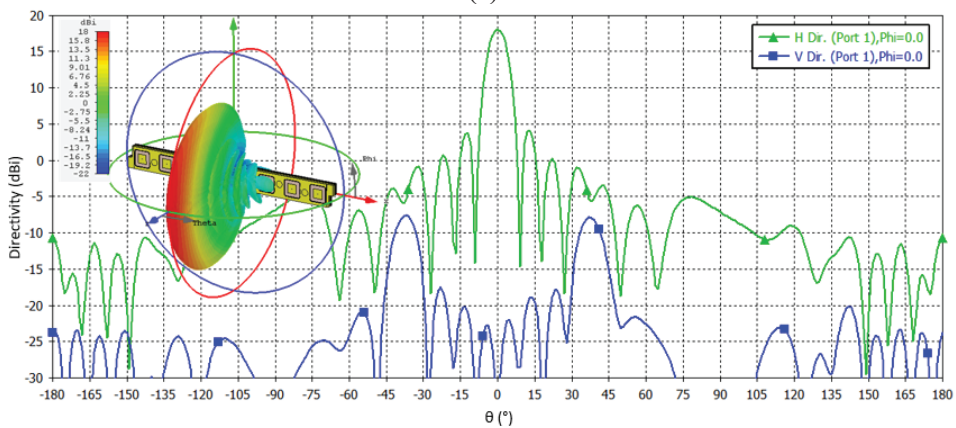

(b)

Figure 12. Simulated radiation patterns (directivity) of the AES shown in Figure 10 at $9.6 \mathrm{GHz}$ in the horizontal plane: (a) port $\mathrm{H}$ and (b) port $\mathrm{V}$ excited. Insets: $3 \mathrm{D}$ views of the radiation patterns.

\section{CONCLUSIONS}

The advances in design and fabrication of a miniaturised photonic front-end for the next generation of space SAR applications has been reported which incorporates new features such as centralised signal processing and a truly broadband frequency opera tion ap proach thanks to the multi-beam TTD reconfigurable bea mforming architecture. 


\section{ACKNOWLEDGEMENTS}

This work was supported by EU-funded H2020 project RETINA under grant agreement n ${ }^{\circ} 821943$.

\section{REFERENCES}

[1] Elachi C.,T. Bicknell, R. Jordan, and C. Wu, Spaceborne Synthetic Aperture Imaging Radars: Application, Techniques, and Technology, IEEE Vol. 70, October 1982

[2] A. Reigber et al., "Very-High-Resolution Airborne Synthetic Aperture Radar Imaging: Signal Processing and Applications," in Proceedings of the IEEE, vol. 101, no. 3, pp. 759-783, March 2013.

[3] Bauters, J. F., Heck, M. J. R., John, D., Dai, D., Tien, M. C., Barton, J. S., Leinse, A., Heideman, R. G., Blumenthal, D. J. and Bowers, J. E., " Photonic Front-End for the next-generation of space SAR applications," Opt. Express, vol. 19, pp. 3163-3174, February 14, (2011)

[4] A. Brimont, D. Zurita, V. C. Duarte, T. Mengual, B. Chmielak, S. Suckow, A. Giesecke, M. A. Piqueras, P. Sanchis, "Optical fiber-to-chip assembly process for ultra-low loss photonic devices based on silicon nitride for space applications", European Conference on Integrated Optics (ECIO), Online, (2020).

[5] M. A. Piqueras, T. Mengual, B. Chmielak, A. Catalani, P. Huggard, H. Wang, R. Ortuño, " Photonic Front-End for the next-generation of space SAR applications," SPIE Remote Sensing, 2015, Toulouse, France

[6] Lumerical: High-Performance Photonic Simulation Software, https://www.lumerical.com/ 